\title{
Geochemical modelling from benchtop to field scale
}

\author{
D. KIRSTE ${ }^{1 *}$, L.M. VOLDEN ${ }^{2}$, J. PEARCE ${ }^{3}$, G. DAWSON ${ }^{3}$ \\ AND S. GOLDING ${ }^{3}$ \\ ${ }^{1}$ Dept. of Earth Sciences, Simon Fraser University, Burnaby, \\ BC, Canada, dkirste@sfu.ca \\ ${ }^{2}$ SRK Consulting Ltd., Vancouver, BC, Canada, \\ lvolden@srk.com \\ ${ }^{3}$ School of Earth and Environmental Sciences, University of \\ Queensland, Brisbane, QLD 4072 Australia: \\ i.pearce2@uq.edu.au, g.dawson@uq.edu.au, \\ s.golding1@uq.edu.au
}

From resource extraction to waste storage and disposal, our transactions with the environment have the potential to pose risks to human and environmental health. Reactive transport models can be useful tools in the assessment, prediction and mitigation of the environmental impacts. In order to generate models, physical and chemical characterization in conjunction with benchtop and field experiments are undertaken to populate and test their viability. These models are a simplification of the complex systems that are being addressed. In the process of simplificaton, decisions are made on what is relevent and important and what can be ignored. However, once the models of the experiments are upscaled to the dimensions and complexities of the real systems, what and how things were simplified can have significant impacts on the predictive output of the models. Mineral precipitation/dissolution, element mobility, and impacts of processes on element mobility are common objectives. To achieve these, elemental accounting including detailed bulk chemistry, mineralogy, mineral composition, and exchange and adsorption site amounts and occupancy is required. With this data, benchtop experiments can be designed. First with batch type reactors, and second, with flow through columns. Reaction path modelling enables establishing the chemical processes and rates derived from batch experiments. Simplifications regarding redox state and reactions, mineral compostion, and reaction rates or equilibrium are applied for the static system. From there, column experiments provide information on the impacts of heterogeneity, coupled flow and transport processes and upscaling. In these, additional simplifications have to be applied and tested, especially those regarding scaling from the static to the dynamic. When upscaling to field conditions, decisions have to account for increases in heterogeneity and the simplifications applied during the developing of the model have to be revisited and justified. Examples from studies on $\mathrm{CO}_{2}$ storage and acid rock drainage will be used to demonstrate how the process evolves. 